\title{
Free-carrier-assisted mid-infrared microcavity soliton generation
}

\author{
Fan, Weichen; Sun, Qibing; Lu, Zhizhou; Wang, Leiran; Zhao, Wei; Zhang, Wenfu
}

Published in:

Journal of Applied Physics

Link to article, DOI:

$10.1063 / 5.0037921$

Publication date:

2021

Document Version

Peer reviewed version

Link back to DTU Orbit

Citation (APA):

Fan, W., Sun, Q., Lu, Z., Wang, L., Zhao, W., \& Zhang, W. (2021). Free-carrier-assisted mid-infrared microcavity soliton generation. Journal of Applied Physics, 129(8), [083106]. https://doi.org/10.1063/5.0037921

\section{General rights}

Copyright and moral rights for the publications made accessible in the public portal are retained by the authors and/or other copyright owners and it is a condition of accessing publications that users recognise and abide by the legal requirements associated with these rights.

- Users may download and print one copy of any publication from the public portal for the purpose of private study or research.

- You may not further distribute the material or use it for any profit-making activity or commercial gain

- You may freely distribute the URL identifying the publication in the public portal

If you believe that this document breaches copyright please contact us providing details, and we will remove access to the work immediately and investigate your claim. 


\title{
Free-carrier-assisted mid-infrared microcavity soliton generation
}

Weichen Fan, 1, 2, a) Qibing Sun, ${ }^{1,2}$ Zhizhou Lu, ${ }^{1,2}$ Leiran Wang, 1,2, b) Wei Zhao, ${ }^{1,2}$ and Wenfu Zhang ${ }^{1,2, c)}$

${ }^{1)}$ State Key Laboratory of Transient Optics and Photonics, Xi'an Institute of Optics and Precision Mechanics,

Chinese Academy of Sciences, Xi'an 710119, China

${ }^{2)}$ University of Chinese Academy of Sciences, Beijing 100049, China

(Dated: 23 January 2021)

\begin{abstract}
Multi-photon absorption (MPA) and free-carrier (FC) effects, usually considered to be detrimental to microcomb generation by introducing strong nonlinear loss, also offer opportunities to overwhelm the thermal-optic effect by modifying the refractive index. Here, we derive the theoretical expression of solitons expanded with MPA and FC effects, in accompany with numerical simulations to reveal the dynamics and mechanism for capturing steady mid-infrared solitons in both $\mathrm{Si}$ and Ge microcavities. It is found that with increased FC lifetime, the intensity-dependent MPA underlies non-monotonous variation of nonlinear detuning which enables soliton generation. More interestingly, proper control on the FC lifetime admits bidirectional switching of soliton states (i.e., both decreasing and increasing the number of solitons) as well as self-starting solitons, yielding different technique routes towards microcavity solitons. This research could contribute to a better understanding of nonlinear behaviors influenced by FC effects, and find practical applications by releasing the demand on precise control of laser sources, which is especially meaningful for the mid-infrared region.
\end{abstract}

\section{INTRODUCTION}

Temporal solitons ${ }^{1-5}$ in microcavities resulting from the double balance between parametric gain and cavity loss together with dispersion and nonlinearity ${ }^{1}$, are preferred versatile light sources for extensive applications including high-precision spectroscopy ${ }^{6}$, optical communications ${ }^{7}$, optical imaging ${ }^{8}$, microwave photonics ${ }^{9}$, and ultrafast range measurement ${ }^{10,11}$ due to their advantages of compactness, robustness and broad bandwidth. To enable microresonatorbased frequency combs accessing the low-noise soliton regime, various approaches have been proposed ${ }^{12-16}$. For example, the fast pump frequency scanning method was first experimentally demonstrated ${ }^{12}$, through which the intracavity field can convert into the soliton operation from the modulation instability (MI) state by tuning the pump frequency from the blue- to red-detuned region. Single-soliton generation can also be achieved via the thermal tuning approach, by changing the driven current (or voltage) on an integrated heater to control the resonance wavelength ${ }^{13}$. In addition, the "power kicking"14, auxiliary-laser-heating ${ }^{15}$ and self-injection locking ${ }^{16}$ all have been proposed as well. Apart from single solitons, other different states such as soliton crystals $^{17-20}$ and breathing solitons ${ }^{21,22}$, have also been successfully obtained. Generally, aforementioned methods require precise control on either pump frequency or operation temperature (and their tuning speeds) to maintain soliton states, and thus become technically challenging in practice, especially for those wavelength ranges such as the midinfrared (MIR) where performances of laser sources are rather limited.

For the MIR region, silicon (Si) and germanium $(\mathrm{Ge})$ are

\footnotetext{
${ }^{a)}$ Present Address: Department of Photonics Engineering, Technical University of Denmark, Kgs. Lyngby, 2800, Denmark

b) Electronic mail: lionking@ opt.ac.cn

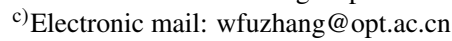

usually considered as promising material platforms for soliton generation since they possess large Kerr nonlinearity as well as high refractive index, which are beneficial for lowthreshold and broadband operation. However, both of them are centrosymmetric materials, which means that $\mathrm{Si}$ and $\mathrm{Ge}$ microcavities do not allow the second order nonlinearities and can hardly be tuned by the Pockels effect ${ }^{23}$. Moreover, they suffer the MPA in the near- and mid-infrared regime, introducing FCs which can inhibit soliton formation since they cause additional loss ${ }^{24,25}$. On the other hand, further study shows that with a positive-intrinsic-negative (PIN) structure, the FC lifetime can be changed by a reverse bias voltage ${ }^{26}$. Numerical investigations also suggest that by properly controlling the FC lifetime, microcombs can reach the multi-soliton state without linear frequency-detuning process, which enables rapid electrical modulation on microresonators ${ }^{27}$. Such principle has been experimentally verified in the Si microring recently ${ }^{28}$. These achievements could provide a potential route for microcavity soliton generation, whereas detailed mechanism and dynamics are still under exploration. Furthermore, the interplay between Kerr and FC effects can lead to new physics and phenomena, which are meaningful to achieve better description and manipulation of soliton behaviors.

In this paper, the mechanism and dynamics of soliton generation in $\mathrm{Si}$ and $\mathrm{Ge}$ microcavities with MPA, FC absorption (FCA) and FC dispersion (FCD) are investigated by deriving the approximate theoretical solution and numerical simulations. It is found that the FCD can introduce an intensitydependent nonlinear detuning which enables the optical field accessing a steady soliton state through three different routes. Soliton generation process via FC lifetime tuning exhibits characteristic step-like patterns, and MPA leads to the distinct physical mechanism, that is, the soliton step is generated through non-monotonously sweeping rather than constantly increasing the nonlinear detuning. Furthermore, bidirectional switching of soliton states and self-starting soliton generation are realized with appropriate FC lifetime and pump parameters, which could allow triggering soliton generation directly in the red-detuning region. These results could not only help 
to a deeper understanding of FC-assisted soliton formation dynamics, but also provide a versatile method to achieve microcavity solitons on centrosymmetric material platforms suffering from MPA and FC effects especially for the mid-infrared region.

\section{THEORETICAL MODEL}

An extended Lugiato-Lefever equation (LLE) considering both the MPA and FC effects is written in the form of the Landau-Euler equation to derive the approximate theoretical solution of solitons ${ }^{29}$ :

$$
\begin{gathered}
\frac{\delta \mathscr{L}}{\delta A^{*}}=\frac{\partial \mathscr{L}}{\partial A^{*}}-\frac{\partial}{\partial \tau} \frac{\partial \mathscr{L}}{\partial\left(\partial A^{*} / \partial \tau\right)}-\frac{\partial}{\partial t} \frac{\partial \mathscr{L}}{\partial\left(\partial A^{*} / \partial t\right)}=\mathscr{R}, \\
\mathscr{L}=i \frac{t_{R}}{2}\left(A^{*} \frac{\partial A}{\partial t}-A \frac{\partial A^{*}}{\partial t}\right)-\frac{\beta_{2} l}{2}\left|\frac{\partial A}{\partial \tau}\right|^{2}+\frac{\gamma l}{2}|A|^{4} \\
\quad-\delta_{0}|A|^{2}, \\
\mathscr{R}=-i \alpha A+i \sqrt{\kappa P_{i n}}-i \frac{\beta_{2 P A} l}{2 A_{\text {eff }}}|A|^{2} A-i \frac{\beta_{3 P A} l}{3 A_{\text {eff }}^{2}}|A|^{4} A \\
\quad-i \frac{\beta_{4 P A} l}{4 A_{\text {eff }}^{3}}|A|^{6} A-\frac{\sigma l}{2}(i-\mu) N_{c} A,
\end{gathered}
$$

where $\mathscr{L}$ is the Lagrangian density, $\mathscr{R}$ is the perturbation term, $t_{R}$ is the round-trip time, $A$ is the intracavity field, $l$ is the cavity length, $\beta_{2}$ is the group velocity dispersion (GVD) coefficient, $\gamma$ is the nonlinear coefficient, $t$ describes the slow time, $\tau$ describes the fast time, $\alpha=\left(\alpha_{0} l+\kappa\right) / 2$ contains the linear propagation and coupling loss, $\delta_{0}$ is the linear detuning, $A_{\text {eff }}$ is the effective mode area, $\sigma$ denotes the FCA crosssection, $\mu$ denotes the FCD coefficient, and $\kappa$ corresponds to the coupling coefficient of the pump power $P_{i n}$. $\beta_{n P A}$ denotes the MPA coefficient, where $n=2,3,4$ corresponds to the two-, three-, and four-photon absorption, respectively. The FC density $N_{c}$ can be described by the rate equation ${ }^{30}$ :

$$
\begin{gathered}
\frac{\partial N_{c}}{\partial t}=\frac{\beta_{2 P A}}{2 \hbar \omega_{p}} \frac{\left\langle|A|^{4}\right\rangle}{A_{e f f}^{2}}+\frac{\beta_{3 P A}}{3 \hbar \omega_{p}} \frac{\left\langle|A|^{6}\right\rangle}{A_{e f f}^{3}}+\frac{\beta_{4 P A}}{4 \hbar \omega_{p}} \frac{\left\langle|A|^{8}\right\rangle}{A_{e f f}^{4}}-\frac{N_{c}}{\tau_{c}}, \\
\left\langle|A|^{2 n}\right\rangle=\frac{1}{t_{R}} \int_{-\frac{t_{R}}{2}}^{\frac{t_{R}}{2}}|A|^{2 n} d \tau .
\end{gathered}
$$

Here, $\hbar$ is the reduced Planck constant, $\omega_{p}$ is the pump frequency, and $\tau_{c}$ is the FC lifetime. For simplification, we take the 3PA as an example in the following calculation, which is reasonable because there is only one dominant MPA at a certain wavelength. By setting ansatz as $A=B \operatorname{sech}(B \tau / m) e^{i \phi_{0}}$ and employing Euler-Lagrange equation, the approximate theoretical solution for the single-soliton state is given as follows:

$$
\begin{gathered}
\frac{d B}{d t}=-\frac{2 \alpha B}{t_{R}}-\frac{16}{45} \frac{\beta_{3 P A} l B^{5}}{t_{R} A_{e f f}^{2}}-\frac{\sigma l N_{c} B}{t_{R}}+\frac{\pi \sqrt{\kappa P_{i n}}}{t_{R}} \cos \phi_{0} \\
\frac{d \phi_{0}}{d t}=-\frac{\beta_{2} l B^{2}}{2 t_{R} m^{2}}+\frac{\gamma l B^{2}}{t_{R}}-\frac{\delta_{0}}{t_{R}}-\frac{\sigma l \mu N_{c}}{t_{R}}
\end{gathered}
$$

where $\phi_{0}$ and $B$ denote the phase and amplitude of the soliton, respectively, and $m$ is a constant ${ }^{31}$. For the stationary state $(\partial / \partial t=0)$, the FC density can be calculated as:

$$
N_{c}=\frac{\beta_{3 P A}}{3 \hbar \omega_{p}} \frac{\left\langle|A|^{6}\right\rangle}{A_{e f f}^{3}} \tau_{c} .
$$

Equations (6) and (7) illustrate the physical mechanisms of FCA and FCD. For analysis, they are regarded as polynomials in terms of $B$. From eq. (6) it can be seen that both FCA and linear cavity loss are the coefficients of the linear term, which means that the impact of FCA is equivalent to the linear cavity loss, possibly prohibiting soliton generation. In the same way, it can be found from eq. (7) that the influence of FCD is similar with the linear cavity detuning. According to eq. (7), FCs can introduce additional nonlinear detuning $\delta=\sigma l \mu N_{c} / 2$ that enables frequency combs which are at the modulation instability (MI) state $\left(d \phi_{0} / d t \neq 0\right.$, Fig. 1 (a) (ii)), to reach the steady soliton state $\left(d \phi_{0} / d t=0\right.$, Fig. 1 (a) (iii)), which is in agreement with previous results ${ }^{27,28}$. As shown in Fig. 1 (a), this nonlinear detuning shifts the cavity resonance to higher frequency, opposite to the direction caused by Kerr and thermo-optic effects ${ }^{12,32}$. Therefore, it could leverage FC effects to compensate or overwhelm the Kerr and thermal effects, which underlies the bidirectional switching of soliton states and self-starting soliton generation as demonstrated in the following sections.

\section{SOLITON GENERATION IN THE SILICON MICROCAVITY}

\section{A. Route 1: FC Lifetime Tuning}

To study the FC-assisted soliton generation process, $\mathrm{Si}$ and Ge microresonators are designed with optimized dispersion and experimentally reasonable structures (see Appendix Table I for parameters). $3.0 \mu \mathrm{m}$ and $4.2 \mu \mathrm{m}$ correspond to the absorption of $\mathrm{HCl}$ and $\mathrm{CO}_{2}$ molecules, respectively, thereby the generated solitons have potential applications in molecular spectroscopy. The thermal effect is not contained in the simulation for simplification since it could be suppressed by using a thermal electrical cooler to stabilize the temperature of microcavities. By solving the LLE accompanied with the rate equation (eqs. (1)-(4)), detailed soliton generation process in the Si microcavity via FC lifetime tuning can be obtained as depicted in Fig. 1. Firstly the pump is tuned from the blue-detuning into the red-detuning region to generate the microcomb at MI state, with FC lifetime $\tau_{c}$ kept at $15 \mathrm{ps}$. 

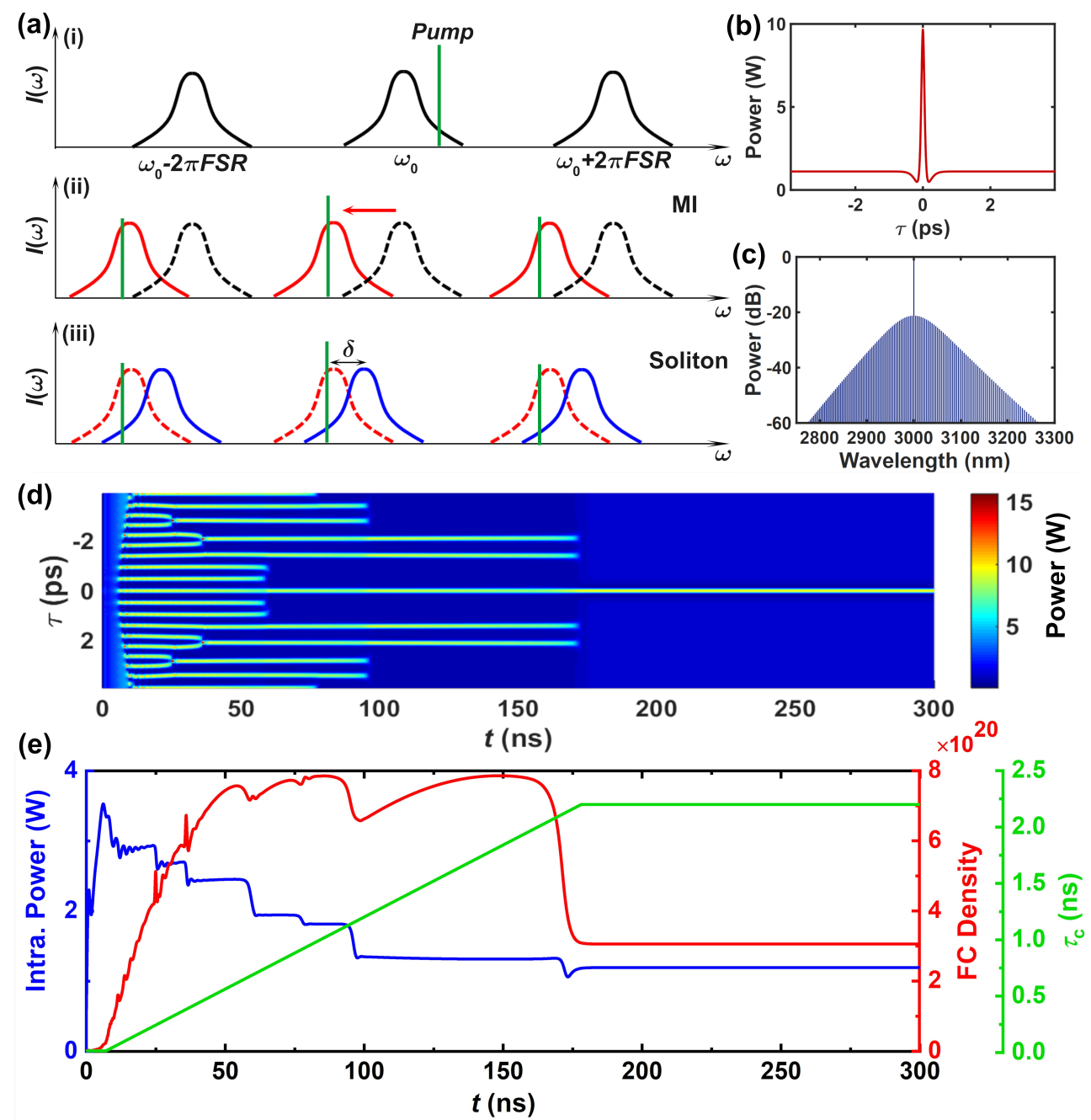

FIG. 1. (a) Schematic interplay between the Kerr, thermo-optic and FC effects. (i) Initial resonance frequency. (ii) Redshift of the resonance induced by the Kerr and thermo-optic effects. (iii) Blueshift of the resonance induced by the FCD. (b)-(e) Soliton generation via FC lifetime tuning in the Si microresonator with pump of $50 \mathrm{~mW}$. (b) Temporal and (c) spectral profiles of the single-soliton state. Evolutions of (d) the temporal field, as well as (e) intracavity power (blue) and FC density (red) along with increasing $\tau_{c}$ from 15 ps to 2.2 ns (green).

Then $\tau_{c}$ is linearly increased to $2.2 \mathrm{~ns}$ at a rate of $0.1 \mathrm{ps}$ per round-trip along with fixed pump. Finally, the $\operatorname{sech}^{2}$-shape temporal and spectral profiles can be obtained (Fig. 1 (b) and (c)), indicating that the microcomb accesses the steady singlesoliton state. Although similar approach has been carried out experimentally ${ }^{28}$, detailed dynamics and mechanisms behind of such process are still yet-to-be revealed so far.

Figures 1 (d) and (e) show corresponding evolutions of the temporal field, intracavity power and FC density. It is worth to note that the FC density does not increase constantly but varies non-monotonously along with the increment of $\tau_{c}$, as shown in Fig. 1 (e). The reason could be explained by eq. (8), wherein it can be found that the FC density is determined both by the optical field intensity and FC lifetime. At the initial MI stage, increasing $\tau_{c}$ enlarges the nonlinear detuning that enables the microcomb reaching multi-soliton state. In the soliton region, for each soliton step the intracavity power is almost unchanged, thus the FC density is proportional to $\tau_{c}$. However, when the microcomb transits from one soliton state into another, the optical field intensity dramatically decreases, leading to the decrement of FC density. Finally, the single soliton can be obtained when the $\tau_{c}$ is kept at $2.2 \mathrm{~ns}$. Besides, as shown in Fig. 1 (e), there is an upper limit on the FC density during this process, which may be because if the FC density exceeds this value, the parametric gain cannot balance the total cavity loss. In this case, the soliton state cannot be sustained. As the $\tau_{c}$ can be the easily and flexibly tuned by varying the applied reverse bias voltage on the $\mathrm{PIN}^{26}$, such process exhibits excellent feasibility and robustness for practical applications.

\section{B. Route 2: Bidirectional Switching of Soliton States}

In most cases, the number of solitons can only be monotonically decreased ${ }^{12-15}$. However, by appropriate control of 
(a)
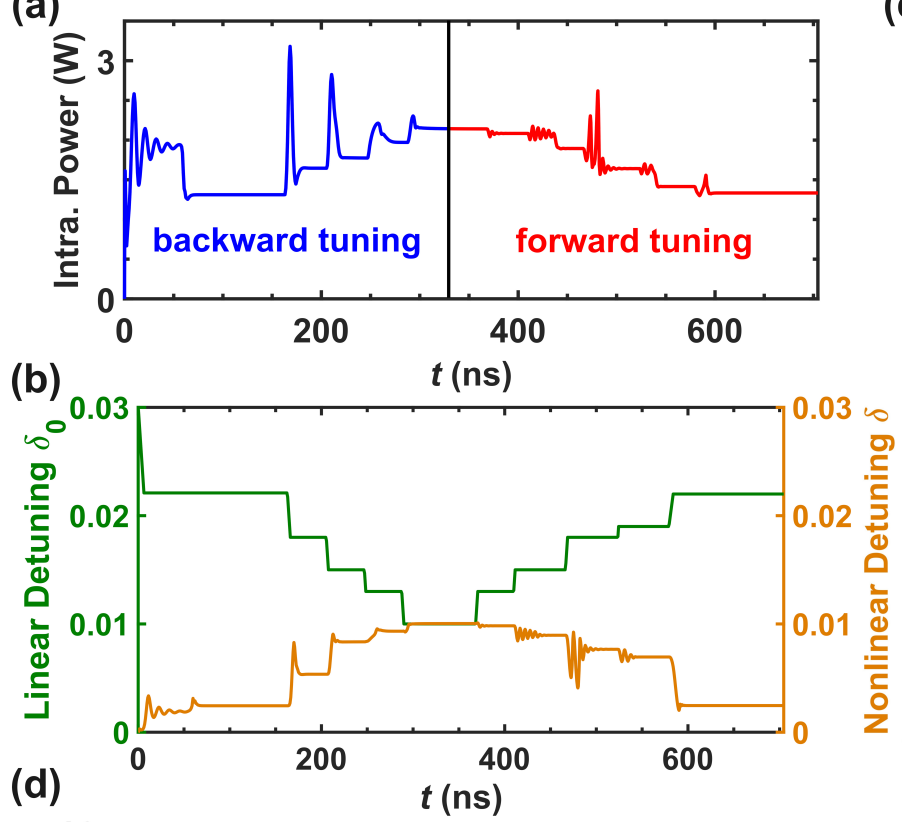

(c)

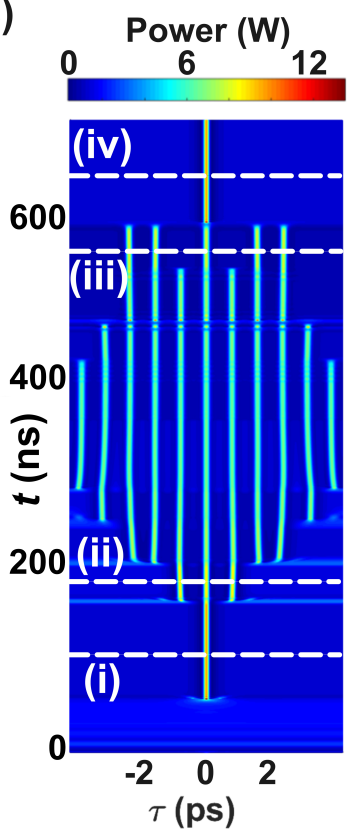

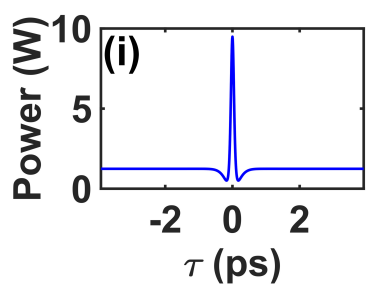
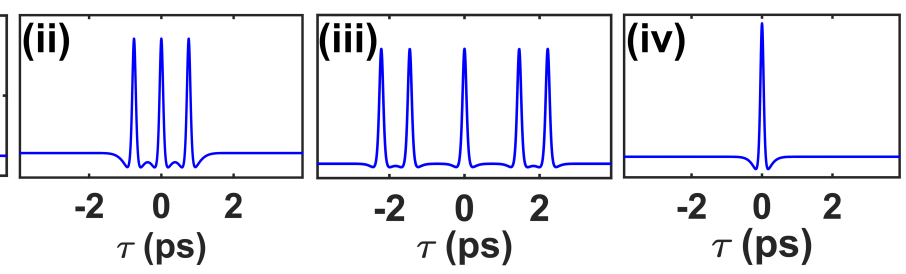

FIG. 2. Bidirectional switching of soliton states in the Si microresonator with $\tau_{c}=2.5 \mathrm{~ns}$ and $P_{\text {in }}=50 \mathrm{~mW}$. (a), (b) Evolutions of intracavity power as well as linear and nonlinear detuning. (c) Temporal evolutions with corresponding temporal profiles shown in (d): (i) single-soliton and (ii) multi-soliton states during backward tuning, as well as (iii) multi-soliton and (iv) single-soliton states during forward tuning.
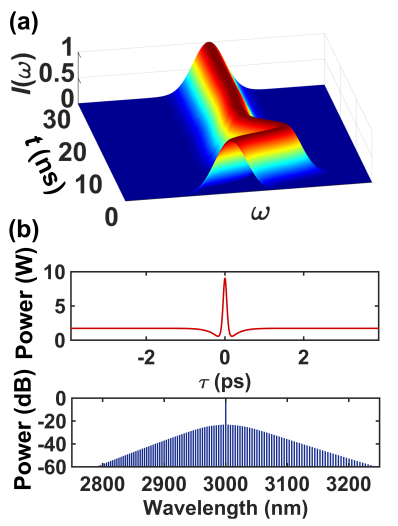

(c)

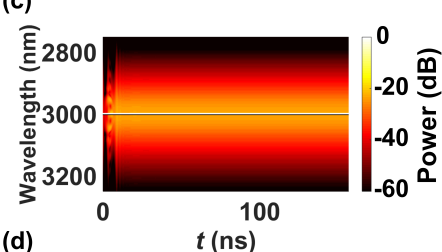

(e)

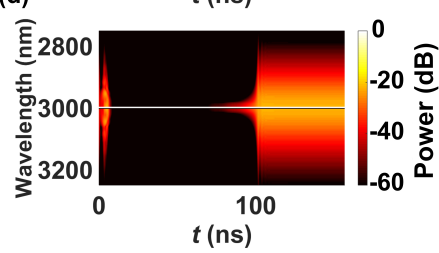

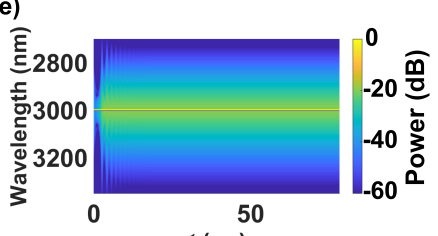

(f) $t(n s)$

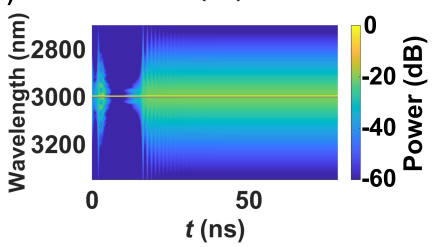

FIG. 3. Self-starting soliton generation in the Si microresonator and influences of FC lifetime and pump power. (a) Schematic shift of the cavity resonance induced by nonlinear detuning during the soliton formation process. (b) Temporal (red) and spectral (blue) profiles for the single-soliton state with $\tau_{c}=3 \mathrm{~ns}$ and $P_{i n}=50 \mathrm{~mW}$. (c)-(f) Spectral evolutions for the condition of (c) $\tau_{c}=3 \mathrm{~ns} \&$ (d) $\tau_{c}=5 \mathrm{~ns}$ with $P_{i n}=50 \mathrm{~mW}$, (e) $P_{i n}=80 \mathrm{~mW} \&$ (f) $P_{i n}=100 \mathrm{~mW}$ with $\tau_{c}=1.5 \mathrm{~ns}$.

the FC lifetime, the FCD-induced nonlinear detuning can balance the Kerr tilt, providing a method for increasing the number of solitons via backward tuning (increasing the pump frequency), as demonstrated in Fig. 2. Figures 2 (a) and (b) show the evolutions of intracavity power as well as linear and nonlinear detuning versus $t$, while the temporal evolution is shown in Fig. 2 (c) and temporal profiles at the four marked states are depicted in Fig. 2 (d). The backward and forward frequency scanning is suspended at each soliton state in order to clearly manifest the soliton steps. Firstly, the single 


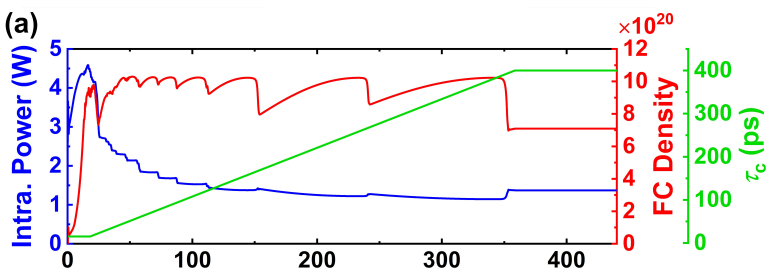

(a)

(b)

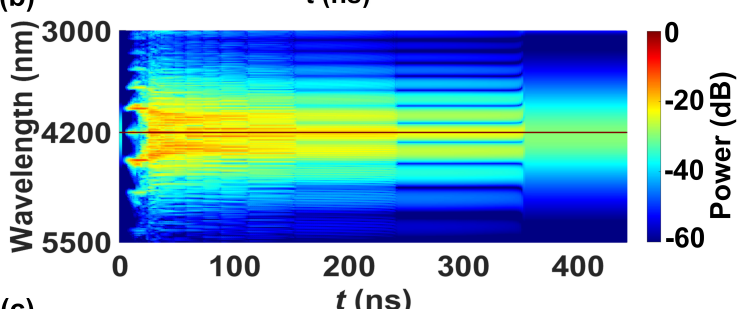

(c)

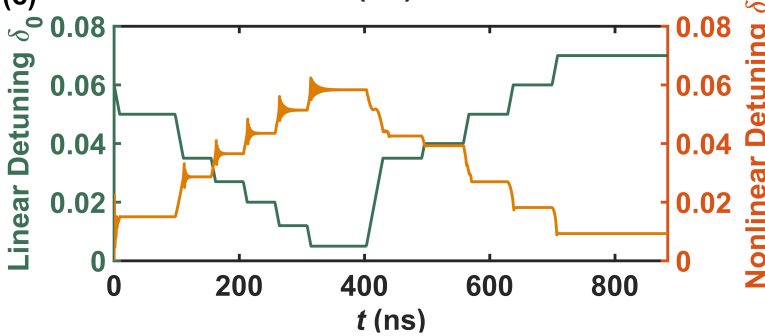

(d)

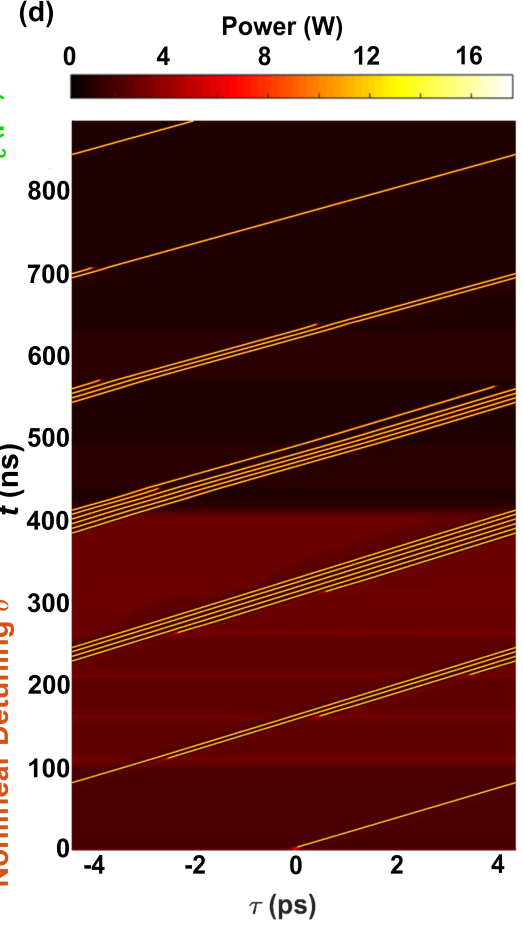

FIG. 4. Soliton generation and bidirectional switching of soliton states in the Ge microresonator with $P_{\text {in }}$ of $200 \mathrm{~mW}$. (a) Soliton steps (blue) and evolution of FC density (red) by tuning $\tau_{c}$ from 15 ps to 400 ps (green). (b) Corresponding spectral evolution indicates that the microcomb reaches the single-soliton state in the end. (c), (d) Evolutions of linear and nonlinear detuning, as well as temporal fields during the bidirectional switching process, with $\tau_{c}=250 \mathrm{ps}$.

soliton is generated by tuning the pump into the resonance from the red side (stage (i)). Further enlarging the pump frequency, it can be seen that the intracavity power gradually increases along with increasing number of solitons (e.g., stage (ii)). The nonlinear detuning increases as well but intervals between each state become smaller and smaller, because it is hard to further accumulate FCs when the density is at a relatively high level. The spike at each transition point is attributed to the delayed response of the $\mathrm{FCD}^{33}$. That is, the intracavity power firstly increases along with the reduction in the linear detuning, and then decreases due to the nonlinear detuning increased by FC accumulation. After the nonlinear detuning is almost unchanged with further decreasing linear detuning, the pump is scanned to lower frequency, and the microcomb converts from the multi-soliton state (e.g., stage (iii)) into the single-soliton state (stage (iv)). In contrast to previous soliton generation wherein the pump is started from blue-detuning region and the number of solitons is unidirectionally tuned ${ }^{12}$, this method can be used to directly generate solitons in the red-detuning region and facilitate bidirectional switching of soliton states.

\section{Route 3: Self-starting Soliton Generation}

With the property to generate solitons bidirectionally, FC effects could also be used for self-starting soliton generation without the need of pump frequency scanning and FC lifetime tuning, as demonstrated in Fig. 3. The pump is set in the reddetuning region with $P_{i n}=50 \mathrm{~mW}$ and $\delta_{0}=0.02$, while the FC lifetime is set as $3 \mathrm{~ns}$ to enhance the influences of nonlinear detuning. Figure 3 (a) shows the schematic shift of the cavity resonance during the soliton generation process. Initially the accumulation of FCs enlarges nonlinear detuning, resulting in the blueshift of cavity resonance. Then the decrease of the intracavity power reduces the nonlinear detuning in turn, which is similar to backward tuning. In the end the intracavity power and the FC density come to the balance, which enables the microcomb reaching the steady single-soliton state, as shown in Fig. 3 (b).

For more detailed soliton generation dynamics with the existence of FCs, influences of FC lifetime and pump power are further studied. Figures 3 (c) and (d) show the spectral evolutions with $\tau_{c}$ of $3 \mathrm{~ns}$ and $5 \mathrm{~ns}$, respectively, at $P_{i n}=50 \mathrm{~mW}$. Figure 3 (e) depicts the results for $P_{i n}=80 \mathrm{~mW}$ with $\tau_{c}=1.5$ ns, while the situation for $P_{i n}=100 \mathrm{~mW}$ is plotted in Fig. 3 (f). It is found that the microcomb can reach steady singlesoliton state faster with shorter FC lifetime or lower pump power, whereas for longer $\tau_{c}$ or higher $P_{i n}$, the system will go through a transient continuous wave $(\mathrm{CW})$ state and thus requires much longer time for stable soliton emission. The reason is that longer FC lifetime or higher pump power can lead to higher peak density of FCs, resulting in the over detuning between the pump frequency and cavity resonance. Since the pump frequency is off-resonance, the intracavity field will convert from the MI to transient $\mathrm{CW}$ state. Such microcomb 

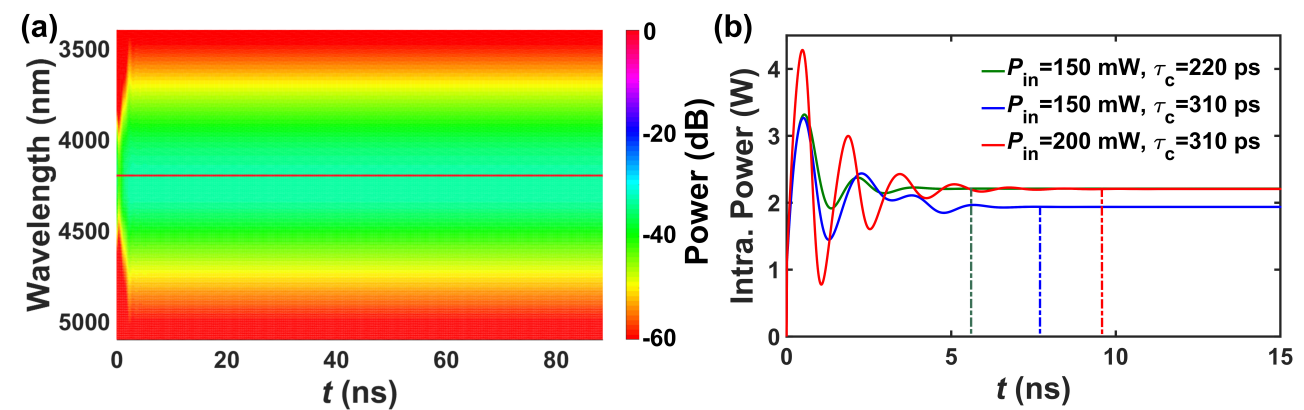

FIG. 5. (a) Spectral evolution for self-starting soliton generation with $\tau_{c}=220 \mathrm{ps}$ and $P_{i n}=150 \mathrm{~mW}$. (b) Evolutions of intracavity power for the conditions of $\tau_{c}=220 \mathrm{ps}$ at $P_{i n}=150 \mathrm{~mW}$ (green), $\tau_{c}=310 \mathrm{ps}$ at $P_{i n}=150 \mathrm{~mW}$ (blue) and $\tau_{c}=310 \mathrm{ps}$ at $P_{i n}=200 \mathrm{~mW}$ (red), respectively.

system favors lower power along with soliton existence in a wider range of $\tau_{c}$, which is beneficial for practical applications by releasing the demand on precise control of both lasers and FC lifetime (e.g., via finely tuning the bias voltage on the PIN).

\section{RESULTS IN THE GERMANIUM MICROCAVITY}

To verify the universality of this FC lifetime manipulation approach, its performance on the Ge platform is also investigated as depicted in Figs. 4 and 5, where phenomena similar with the Si platform are observed. Figures 4 (a) and (b) show that, in a Ge microring the FCD can also transform microcomb operation from the MI to multi-soliton and finally the single-soliton state along with increasing $\tau_{c}$ from 15 ps to 400 ps at a rate of $0.01 \mathrm{ps}$ per round-trip. Due to the large 3PA and FCD coefficient, the needed FC lifetime for stable soliton generation in a Ge microcavity is shorter than that in Si microcavities (i.e., hundreds of picoseconds vs. several nanoseconds level). Bidirectional switching of the soliton number is also achieved in the Ge microcavity, as demonstrated in Figs. 4 (c) and (d). The soliton drift resulting from the third-order dispersion could be compensated by the Raman effect ${ }^{34}$. As presented in Fig. 5 (a), the single soliton can self-start and access the steady state rapidly for $\tau_{c}=220 \mathrm{ps}$ at $P_{\text {in }}$ of $150 \mathrm{~mW}$, while Fig. 5 (b) indicates that the evolution process becomes slower for a longer FC lifetime of $310 \mathrm{ps}$ at $P_{\text {in }}$ of $150 \mathrm{~mW}$ and higher pump power of $200 \mathrm{~mW}$ with $\tau_{c}$ of $310 \mathrm{ps}$, reflecting the same manner as Si microring does.

\section{DISCUSSIONS AND CONCLUSION}

These results suggest that, the FC-assisted soliton formation can be applicable for not only $\mathrm{Si}$ and Ge but also other centrosymmetric material platforms suffering strong MPA. In addition, the soliton "steps" here are intrinsically different but with similar characteristic to those observed via the pump frequency scanning method ${ }^{12}$, which is rational since it can be attributed to the FCD-induced alteration of the total cavity detuning as illustrated above. In the presence of this nonlinear detuning, three different routes to soliton generation are demonstrated, as shown in Fig. 6, which could enable self-starting soliton generation and bidirectional switching of soliton states. This approach could release requirements for high-performance tunable narrow-linewidth lasers, which is especially meaningful for the MIR region. Also it could help to a faster tuning speed than the thermal-tuning approach ${ }^{28}$, thanks to easy controllability of the FC lifetime (e.g., through an integrated PIN structure). With appropriate parameter settings this method could also be utilized in the exploration of extraordinary soliton forms such as soliton crystals, breathers, etc.

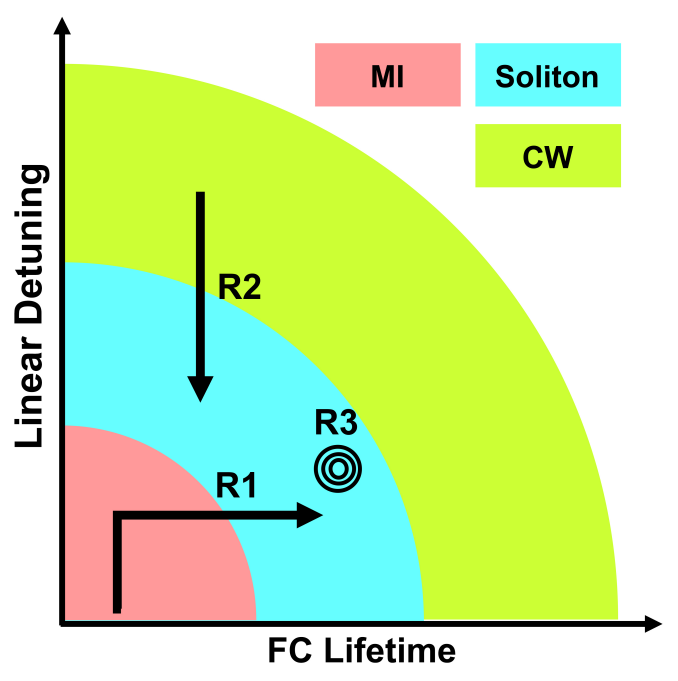

FIG. 6. Three different routes to achieving microcavity solitons in the presence of MPA and FC effects. R1: FC lifetime tuning. R2: Bidirectional switching. R3: Self-starting.

In summary, this work theoretically and numerically illustrates mechanism and dynamics for the FC-assisted soliton generation in $\mathrm{Si}$ and Ge microcavities. An expanded approximate theoretical description containing the MPA, FCA and FCD effects is derived to reveal the complex nonlinear processes. It is found that with the increase of FC lifetime, the intensity-dependent nonlinear detuning varies repeatedly, which enables characteristic soliton steps to be observed, and the microcomb gradually transforms from the MI and multisoliton into the steady single-soliton state. Further investiga- 
tions show that with suitable FC lifetime, bidirectional switching of soliton states and self-starting soliton generation can also be achieved, which could provide diverse ways to trigger microcavity solitons. Such results can help to a deeper understanding of mechanism and dynamics of soliton generation in the presence of FC effects, as well as find important potentials in the development of microcavity soliton techniques.

\section{ACKNOWLEDGMENTS}

The authors thank Hairun Guo and Xiaohong $\mathrm{Hu}$ for valuable discussions. The authors acknowledge the support from the National Natural Science Foundation of China (NSFC) (Grant No. 61635013, 61675231, 61705257, and 61805277), the Strategic Priority Research Program of the Chinese Academy of Sciences (Grant No. XDB24030600), and the Youth Innovation Promotion Association of CAS (No. 2016353).

\section{DATA AVAILABILITY}

The data that support the findings of this study are available from the corresponding author upon reasonable request.

\section{Appendix A: Simulation Parameters}

The effective mode area and dispersion of Si and Ge microcavities are calculated by finite element method.

TABLE I. Parameters of Si and Ge microresonators for simulation

\begin{tabular}{ccc}
\hline \hline & $\mathrm{Si}$ & $\mathrm{Ge}$ \\
\hline Pump Wavelength $(\mu \mathrm{m})$ & 3.0 & 4.2 \\
Cross-section $\left(\mu \mathrm{m}^{2}\right)$ & $1.6 \times 0.5$ & $2.5 \times 1.3$ \\
$A_{\text {eff }}\left(\mu \mathrm{m}^{2}\right)$ & 0.866 & 2.40 \\
Rib Height $(\mu \mathrm{m})$ & 0.2 & 0.2 \\
$\alpha_{0}(\mathrm{~dB} / \mathrm{cm})$ & 0.7 & 2.0 \\
Radius $R(\mu \mathrm{m})$ & 100 & 100 \\
$F S R(\mathrm{GHz})$ & 128 & 113 \\
$\kappa$ & 0.0100 & 0.0289 \\
$\gamma\left(\mathrm{W}^{-1} \mathrm{~m}^{-1}\right)$ & 9.67 & 15.90 \\
$\beta_{2}\left(\mathrm{ps}^{2} / \mathrm{km}\right)$ & -386 & -186 \\
$\beta_{3 P A}\left(\mathrm{~m}^{3} / \mathrm{W}^{2}\right)^{28,35}$ & $2.0 \times 10^{-27}$ & $2.7 \times 10^{-25}$ \\
$\sigma\left(\mathrm{m}^{2}\right)^{28,36}$ & $5.88 \times 10^{-21}$ & $6.39 \times 10^{-22}$ \\
$\mu^{28,36}$ & 3.75 & 116.58 \\
\hline \hline
\end{tabular}

${ }^{1}$ T. J. Kippenberg, A. L. Gaeta, M. Lipson, and M. L. Gorodetsky, Science 361, eaan8083 (2018)

${ }^{2}$ C. Bao, Y. Xuan, D. E. Leaird, S. Wabnitz, M. Qi, and A. M. Weiner, Optica 4, 1011 (2017).

${ }^{3}$ M. Yu, Y. Okawachi, R. Cheng, C. Wang, M. Zhang, A. L. Gaeta, and M. Lončar, Light Sci. Appl. 9, 9 (2020).
${ }^{4}$ Z. Gong, X. Liu, Y. Xu, and H. X. Tang., Optica 7, 1275 (2020).

${ }^{5}$ G. Moille, L. Chang, W. Xie, A. Rao, X. Lu, M. Davanço, J. E. Bowers, and K. Srinivasan, Laser Photonics Rev. 14, 2000022 (2020).

${ }^{6}$ Q. Yang, B. Shen, H. Wang, M. Tran, Z. Zhang, K. Y. Yang, L. Wu, C. Bao, J. Bowers, A. Yariv, and K. Vahala, Science 363, 965 (2019).

${ }^{7}$ B. Corcoran, M. Tan, X. Xu, A. Boes, J. Wu, T. G. Nguyen, S. T. Chu, B. E. Little, R. Morandotti, A. Mitchell, and D. J. Moss, Nat. Commun. 11, 2568 (2020).

${ }^{8}$ C. Bao, M. G. Suh, and K. Vahala, Optica 6, 1110 (2019).

${ }^{9}$ W. Weng, E. Lucas, G. Lihachev, V. E. Lobanov, H. Guo, M. L. Gorodetsky, and T. J. Kippenberg, Phys. Rev. Lett. 122, 013902 (2019).

${ }^{10} \mathrm{M}$. G. Suh and K. J. Vahala, Science 359, 884 (2018).

${ }^{11}$ P. Trocha, M. Karpov, D. Ganin, M. H. P. Pfeiffer, A. Kordts, S. Wolf, J. Krockenberger, P. Marin-Palomo, C. Weimann, S. Randel, W. Freude, T. J. Kippenberg, and C. Koos, Science 359, 887 (2018).

${ }^{12}$ T. Herr, V. Brasch, J. D. Jost, C. Y. Wang, N. M. Kondratiev, M. L. Gorodetsky, and T. J. Kippenberg, Nat. Photonics 8, 145 (2014).

${ }^{13}$ C. Joshi, J. K. Jang, K. Luke, X. Ji, S. A. Miller, A. Klenner, Y. Okawachi, M. Lipson, and A. L. Gaeta, Opt. Lett. 41, 2565 (2016).

${ }^{14}$ V. Brasch, M. Geiselmann, M. H. P. Pfeiffer, and T. J. Kippenberg, Opt. Express 24, 29312 (2016).

${ }^{15}$ Z. Lu, W. Wang, W. Zhang, S. T. Chu, B. E. Little, M. Liu, L. Wang, C. Zou, C. Dong, B. Zhao, and W. Zhao, AIP Adv. 9, 025314 (2019).

${ }^{16}$ B. Shen, L. Chang, J. Liu, H. Wang, Q. Yang, C. Xiang, R. N. Wang, J. He, T. Liu, W. Xie, J. Guo, D. Kinghorn, L. Wu, Q. Ji, T. J. Kippenberg, K. Vahala, and J. E. Bowers, Nature 582, 365 (2020).

${ }^{17}$ D. C. Cole, E. S. Lamb, P. Del'Haye, S. A. Diddams, and S. B. Papp, Nat. Photonics 11, 671 (2017).

${ }^{18}$ W. Wang, Z. Lu, W. Zhang, S. T. Chu, B. E. Little, L. Wang, X. Xie, M. Liu, Q. Yang, L. Wang, J. Zhao, G. Wang, Q. Sun, Y. Liu, Y. Wang, and W. Zhao, Opt. Lett. 43, 2002 (2018).

${ }^{19}$ Z. Lu, W. Wang, W. Zhang, M. Liu, L. Wang, S. T. Chu, B. E. Little, J. Zhao, P. Xie, X. Wang, and W. Zhao, Opt. Mater. Express 8, 2662 (2018).

${ }^{20}$ M. Karpov, M. H. P. Pfeiffer, H. Guo, W. Weng, J. Liu, and T. J. Kippenberg, Nat. Phys. 15, 1071 (2019).

${ }^{21}$ M. Yu, J. K. Jang, Y. Okawachi, A. G. Griffith, K. Luke, S. A. Miller, X. Ji, M. Lipson, and A. L. Gaeta, Nat. Commun. 8, 14569 (2017).

${ }^{22}$ E. Lucas, M. Karpov, H. Guo, M. L. Gorodetsky, and T. J. Kippenberg, Nat. Commun. 8, 736 (2017).

${ }^{23}$ L. Zhang, A. M. Agarwal, L. C. Kimerling, and J. Michel, Nanophotonics 3, 247 (2014).

${ }^{24}$ R. K. W. Lau, M. R. E. Lamont, Y. Okawachi, and A. L. Gaeta, Opt. Lett. 40, 247 (2015).

${ }^{25}$ M. Liu, L. Wang, Q. Sun, S. Li, Z. Ge, Z. Lu, W. Wang, G. Wang, W. Zhang, X. Hu, and W. Zhao, Photon. Res. 6, 238 (2018).

${ }^{26}$ A. C. Turner-Foster, M. A. Foster, J. S. Levy, C. B. Poitras, R. Salem, A. L. Gaeta, and M. Lipson, Opt. Express 18, 3582 (2010).

${ }^{27}$ T. Hansson, D. Modotto, and S. Wabnitz, Opt. Lett. 39, 6747 (2014).

${ }^{28}$ M. Yu, Y. Okawachi, A. G. Griffith, M. Lipson, and A. L. Gaeta, Optica 3, 854 (2016).

${ }^{29}$ A. Sahoo, S. Roy, and G. P. Agrawal, Phys. Rev. A 96, 013838 (2017).

${ }^{30}$ T. Hansson and S. Wabnitz, Nanophotonics 5, 231 (2016).

${ }^{31}$ X. Yi, Q. Yang, K. Y. Yang, and K. Vahala, Opt. Lett. 41, 3419 (2016).

${ }^{32}$ J. R. Stone and S. B. Papp, Phys. Rev. Lett. 125, 153901 (2020).

${ }^{33}$ M. Garcia-Lechuga, J. Siegel, J. Hernandez-Rueda, and J. Solis, J. Appl. Phys. 116, 113502 (2014).

${ }^{34}$ M. Karpov, H. Guo, A. Kordts, V. Brasch, M. H. P. Pfeiffer, M. Zervas, M. Geiselmann, and T. J. Kippenberg, Phys. Rev. Lett. 116, 103902 (2016).

${ }^{35}$ D. Seo, J. M. Gregory, L. C. Feldman, N. H. Tolk, and P. I. Cohen, Phys. Rev. B 83, 195203 (2011).

${ }^{36}$ J. Yuan, Z. Kang, F. Li, X. Zhang, X. Sang, Q. Wu, B. Yan, K. Wang, X. Zhou, K. Zhong, G. Zhou, C. Yu, C. Lu, H. Y. Tam, and P. K. A. Wai, J. Light. Technol. 35, 2994 (2017). 